\title{
A photomultiplier tube model for the water Cherenkov detectors of LAGO
}

\section{J. Peña-Rodríguez, ${ }^{a, *}$ S. Hernández-Barajas, ${ }^{b}$ Y. León-Carreño ${ }^{b}$ and L. A. Núñez ${ }^{a}$ on behalf of the LAGO Collaboration}

(a complete list of authors can be found at the end of the proceedings)

${ }^{a}$ Universidad Industrial de Santander, Escuela de Física

Calle 9 Carrera 27, Bucaramanga, Colombia

${ }^{b}$ Universidad Industrial de Santander, Escuela de Ingeniería Eléctrica, Electrónica y de

Telecomunicaciones

Calle 9 Carrera 27, Bucaramanga, Colombia

E-mail: jesus.pena@correo.uis.edu.co

The Latin American Giant Observatory (LAGO) is an international experiment covering 10 Latin American countries and Spain. LAGO researches gamma-ray bursts and space weather phenomena using water Cherenkov detectors (WCDs) deployed at different altitudes. Large-area (8-inches) photomultipliers sense Cherenkov radiation produced by secondary particles crossing the WCDs. We present a generic photomultiplier model applied to the Hamamatsu R5912 tube, used in most of LAGO' WCDs. The model depends on the number of dynodes, the bias voltage, the number of incident photons, the photodetection efficiency, and the bias network. Besides, the implementation of the model includes a simulation of the front-end of LAGO's acquisition electronics, allowing the linearity of the system to be evaluated under different conditions.

The model was validated with data recorded by the MuTe-Chitaga (Bucaramanga, Colombia) and Nahuelito (Bariloche, Argentina) WCDs. Geant4 simulations estimate the number of Cherenkov photons arriving at the photomultiplier. We contrasted the anode/dynode pulse amplitude ratio between the data and the model prediction. We also compared the estimated and measured vertical equivalent muon charge (pulse area). The vertical-muon-charge estimated by the model (321.6 UADC) differs by $4 \%$ from the measured by the MuTe WCD (333 UADC).

$37^{\text {th }}$ International Cosmic Ray Conference (ICRC 2021)

July 12th-23rd, 2021

Online - Berlin, Germany

\footnotetext{
*Presenter
} 


\section{Introduction}

The LAGO (Latin American Giant Observatory) project consists in single or small arrays of particle detectors at ground level, spanning over different sites, located at several latitudes (from Mexico to Antarctica), different altitudes (from sea level up to more than $5000 \mathrm{~m}$ a.s.1.).

LAGO operates in particle energies ranging from $0.5 \mathrm{GeV}$ to tens of $\mathrm{TeV}$. It detects short transients -like gamma-ray bursts (GRBs) [1] - and long transients -like Forbush decreases- [2-4] by looking for changes in the cosmic ray background using the single-particle counting technique $[5,6]$.

A key point in the LAGO WCDs is the calibration process. We establish a conversion rule from the digitized charge in electronic units to deposited energy in vertical-equivalent muons (VEM) [7, 8]. This relationship depends on the linearity of both the PMT and the electronics readout working together. Thus it is essential to have a PMT model to make the calibration a straightforward process.

Some works tackle modelling of photomultiplier tubes using Spice environments. H. Krihely describes a Spice model which simulates the electron multiplication between inter-dynode stages [9]. The model evaluates the PMT under different bias conditions and voltage divider circuits, but the assumption of a constant bias ratio between the PMT dynodes under no light conditions presents a drawback. This approach simplifies the mathematical process but limits the model robustness and generality for testing under stimulation conditions and voltage divider configurations (i.e. tapered resistive chains).

Akimov et al. propose a computer model of the Hamamatsu R11410-20 photomultiplier based on Spice using current-controlled sources. The authors insert a variable inter-dynode bias fraction and evaluate the limits of the anode current caused by the PMT gain [10]. Hueso-Gonzalez et al. verify Krihely's model compensating some ambiguities and inconsistencies [11].

We propose a general PMT Spice model tuned to the LAGO's PMT (Hamamatsu R5912) parameters. The model adds a variable inter-dynode bias fraction allowing the simulation of different divider circuits (i.e. uniform and tapered). Our PMT model performance is assessed by stimulating with typical photon pulses of LAGO's water Cherenkov detectors. The model evaluates PMT parameters like linearity, transient response, gain, and dynamic range, under different bias and stimulus conditions.

\section{Methods}

\subsection{A generic PMT model}

A PMT is an optoelectronic device that generates a measurable electric current from a luminous signal. The incident photon impinges the photocathode releasing a primary electron. The secondaryelectron emission occurs in three distinct steps: production of internal secondary electrons by the kinetic impact of the primary electron, transport of the secondary electrons through the sample bulk toward the surface, and final escape of the electrons through the solid-vacuum interface. A vacuum glass tube encapsulates all the PMT parts [12]. 
We present a PMT model considering the operation principles and intrinsic parameters (number of amplification stages and gain curve). The total gain of the PMT model is,

$$
G=\frac{I_{a}}{I_{k}}
$$

where $I_{a}$ is the anode current, and $I_{k}$ is the photocathode current.

The PMT gain can be expressed as a function of the gain in each stage,

$$
G=\beta \prod_{i=1}^{N} g_{i}
$$

with $g_{i}$ the gain at each stage, $N$ the number of dynodes, and $\beta$ the collection efficiency. The gain $g_{i}$ depends on the inter-dynode voltage $v_{i}$,

$$
g_{i}=k_{i} v_{i}^{\alpha}
$$

where $k_{i}$ is a constant and $0.6 \leq \alpha \leq 0.8$ is an intrinsic parameter of the PMT. The total gain Eq. 2 can be expressed as the product of all the inter-dynode gains or as function of the PMT bias voltage $V_{B}$,

$$
G=\prod_{i=1}^{N} k_{i}\left(V_{B} \epsilon_{i}\right)^{\alpha},
$$

here $\epsilon_{i}$ is the fraction of the bias voltage in each inter-dynode stage.

The fraction of the bias voltage is defined as

$$
\epsilon_{i}=\frac{R_{i}}{R_{T}}
$$

where $R_{i}$ is the inter-dynode resistance, and $R_{T}$ is the total resistance of the voltage divider.

We assume $k_{i}$ values are equal for all dynodes due its dependence on the dynode material [10], equation Eq. 4 results in

$$
G=k^{N} V_{B}^{N \alpha}\left(\prod_{i=1}^{N} \epsilon_{i}\right)^{\alpha} .
$$

To estimate the value of $\alpha$ and $k$, we define $\varepsilon$ as

$$
\varepsilon=\sqrt[N]{\prod_{i=1}^{N} \epsilon_{i}}
$$

After replacing Eq. 7 in Eq. 6, the gain is

$$
G=k^{N}\left(V_{B} \varepsilon\right)^{N \alpha}
$$




\subsection{Modeling the R5912 PMT}

We extracted a couple of points $\left[V_{B 1}, G_{1}\right]$ and $\left[V_{B 2}, G_{2}\right]$ from the gain curve of the Hamamatsu R5912 PMT to obtain the parameters $\alpha$ and $k$ [13].

The points $\left[1000 \mathrm{~V}, 3 \times 10^{5}\right]$ and $\left[1500 \mathrm{~V}, 7 \times 10^{6}\right]$ were chosen and substitutes in Eq. 8 obtaining a pair of equations which are solved for the unknown variables $(\alpha, k)$.

$$
\begin{aligned}
& G_{1}=k^{N}\left(V_{B 1} \varepsilon\right)^{N \alpha}, \\
& G_{2}=k^{N}\left(V_{B 2} \varepsilon\right)^{N \alpha},
\end{aligned}
$$

where the number of dynodes is $N=10$. The parameter $\varepsilon$ is calculated from the voltage distribution ratio in the resistive polarization chain, provided in the PMT datasheet, as shown in Table 1,

$$
\varepsilon=0.035
$$

Table 1: Tapered voltage distribution of the PMT R5912 for linear measurements [13].

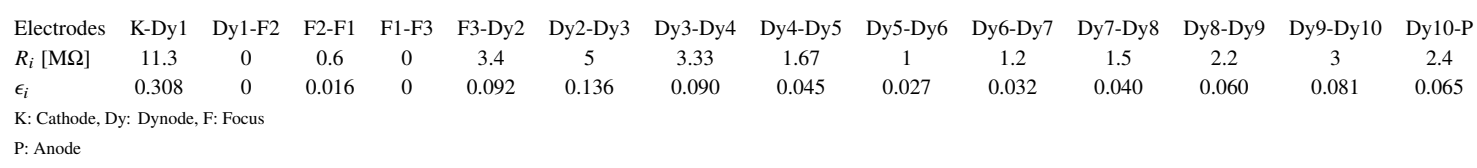

Then, an expression for $k$ is obtained from Eq. 10 as follows,

$$
k=\sqrt[N]{\frac{G_{2}}{\left(V_{B 2} \varepsilon\right)^{N \alpha}}}
$$

and substituting Eq. 12 in Eq. 9 the parameter $\alpha$ is,

$$
\alpha=\frac{\log \left(\frac{G_{1}}{G_{2}}\right)}{N \log \left(\frac{V_{B 1}}{V_{B 2}}\right)} .
$$

From Eq. 12 and Eq. 13 we obtain $k=0.223$ and $\alpha=0.776$.

\subsection{Spice model}

The dynodes and anode currents were modeled as function of the parameters $k, \alpha, \epsilon_{i}, V_{B}$, and $N$. The current flowing through $i$ th dynode is,

$$
I_{d, i}=I_{k} \frac{\left(k V_{B}^{\alpha}\right)^{N}\left(\prod_{i=1}^{N} \epsilon_{i}\right)^{\alpha}}{\left(k v_{i}^{\alpha}\right)^{N+1-i}\left(\prod_{i=1}^{N+1-i} \epsilon_{i}\right)^{\alpha}}, \quad i=1,2, \cdots N
$$

The anode current is,

$$
I_{a}=I_{k} k^{N}\left(V_{B} \varepsilon\right)^{N \alpha} .
$$

The photocathode current $I_{k}$ is,

$$
I_{k}=\frac{Q}{t}
$$


where $Q$ is the electric charge in the photocathode during the time step $t$. The electric charge is,

$$
Q=N_{p e} * e,
$$

with $e$ the electron charge $\left(1.6 \times 10^{-19} \mathrm{C}\right)$.

The PMT model is simulated using the Orcad PSpice software. We used the GVALUE block to model the PMT currents flowing along the PMT dynodes. This block sets the transfer function described by Eq. 14 and Eq. 15 for each amplification stage depending on the voltage applied.

We selected a tapered divider with decoupling capacitors to reduce nonlinearities in the PMT response due to the space-charge effect (large current flowing in the dynodes) in pulse-mode operation $[14,15]$. The resistor values were estimated following the inter-dynode ratios recommendations showed in Table 1.

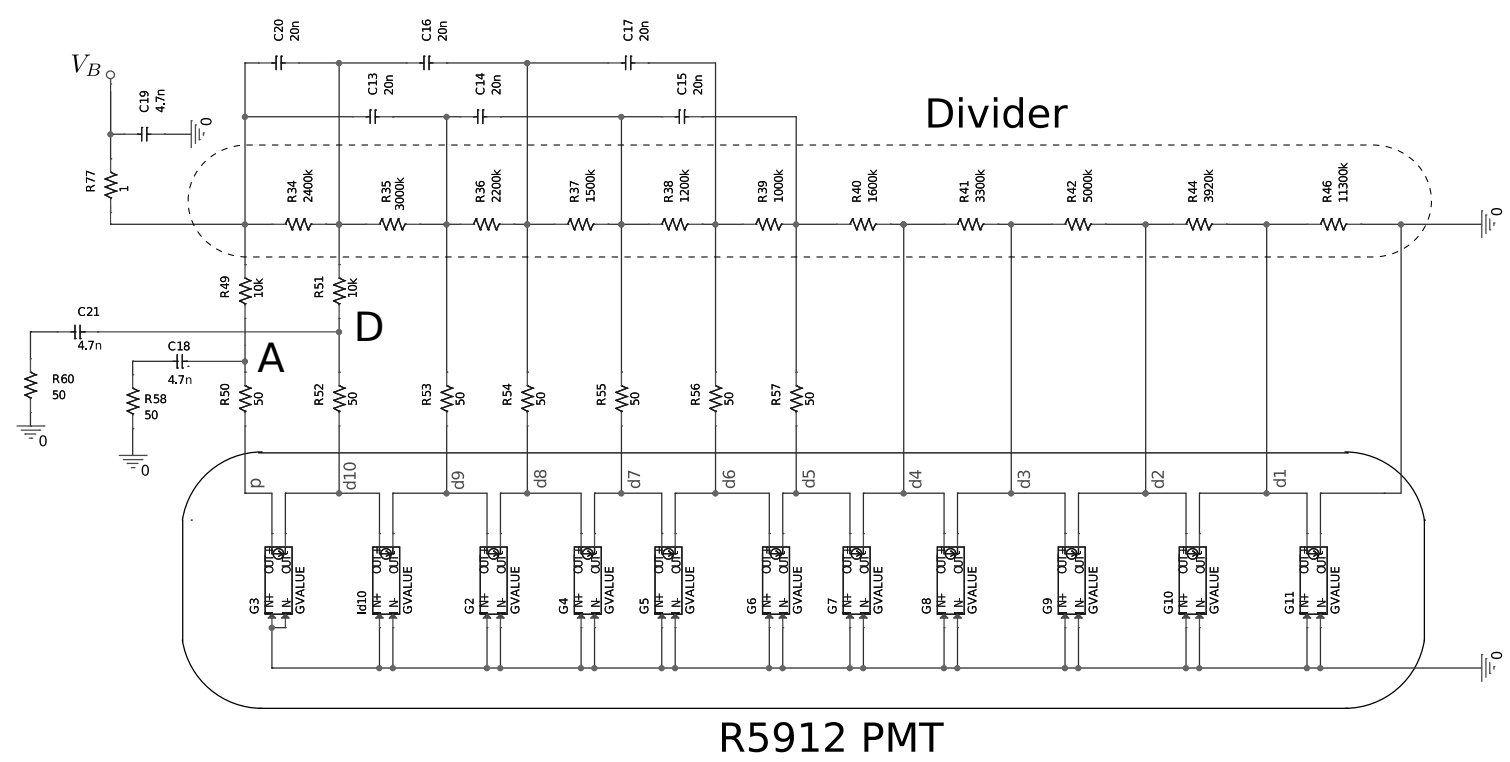

Figure 1: SPICE model of the R5912 PMT (solid-line frame) and the tapered resistive chain (dashed-line frame). The dynode and anode outputs are indicated by A and D respectively.

We installed coupling capacitors of $4.7 \mathrm{nF}(\mathrm{C} 18$ and $\mathrm{C} 21)$ to filter the DC component in the anode and the last dynode output. Output loads of $50 \Omega$ avoid signal reflections due to bad impedance coupling in the transmission lines. Fig. 1 shows the schema of the designed Spice model.

The shape of the photo-electron pulse at the PMT photocathode depends on the arrival time of the incident photons. We used a photo-pulse resulting from $3 \mathrm{GeV}$ vertically crossing muons to a $120 \mathrm{~cm}$ height WCD [16, 17]. The pulse releases around 203 photo-electrons decreasing exponentially with time constant of $\sim 42.12 \mathrm{~ns}$ and a time width (at the $10 \%$ amplitude) of $\sim 100 \mathrm{~ns}$. 


\section{Results}

\subsection{Simulated vertical muon charge}

A $3 \mathrm{GeV}$ vertical muon impinging the WCD releases a photo-cathode current peak of $\sim 17 \mathrm{nA}$. The PMT model was biased at $1000 \mathrm{~V}\left(2.9 \times 10^{5}\right.$ gain). When a vertical muon hits the WCD, a current signal of $\sim 5 \mathrm{~mA}$ is measured at the anode, and a voltage pulse of $\sim 250 \mathrm{mV}$ appears across the load resistance $(50 \Omega)$. The maximum anode dark current (unwanted current resulting from thermally excited electrons) establishes the low boundary of acquisition at $0.7 \mu \mathrm{A}$ [18].
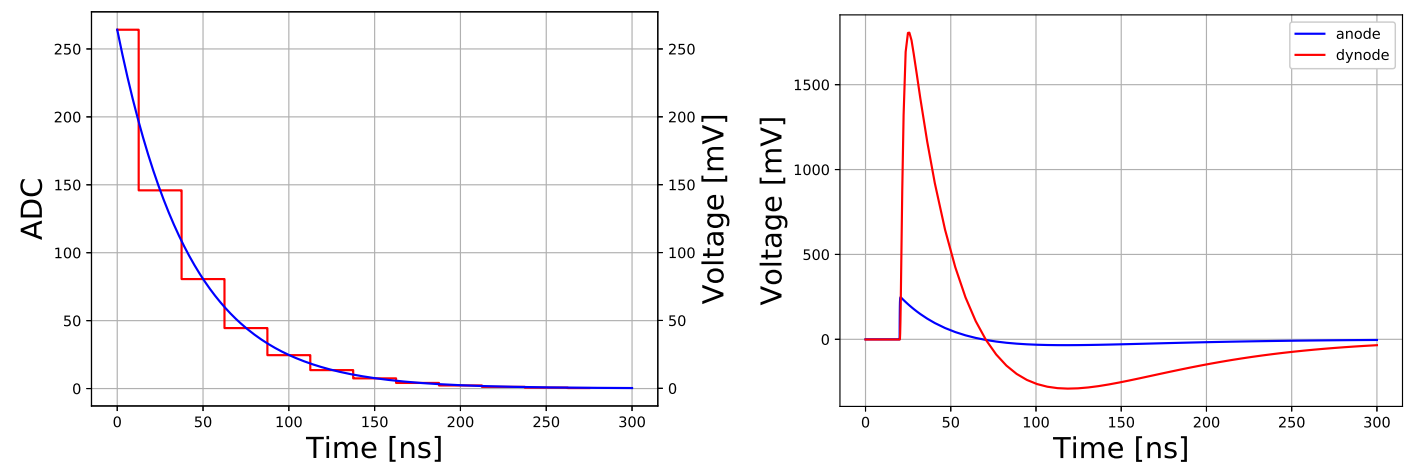

Figure 2: (Left) Pulse shape emulation (blue-line) and digitization (red-line) of a vertical muon signal. The electronics ADC samples/holds the anode voltage every $25 \mathrm{~ns}$, then a 12-bin vector stores the digitized pulse shape. (Right) Anode (blue-line) and amplified dynode (red-line) outputs obtained from the SPICE model at $1000 \mathrm{~V}$ for a photocathode current of $17 \mathrm{nA}$. The dynode/anode ratios is 7.3 and the pulse width is $\sim 50 \mathrm{~ns}$.

The LAGO readout system digitizes the PMT pulses at $40 \mathrm{MHz}$ with a resolution of 10 bits $(1 \mathrm{mV} / \mathrm{UADC})$; the pulse shape is stored in a 12-bin vector $(300 \mathrm{~ns})$ [19]. We emulate the digitization process of the model outputs to compare simulations and data as shown in Fig. 2 (left). The resulting pulse charge of the simulated vertical muon was $321.6 \mathrm{UADC}$, differing about $4 \%$ of the value obtained by the MuTe-LAGO's WCD (333 UADC) [20].

\subsection{Response of the PMT model}

Fig. 2 (right) shows the dynode and anode output for a photocathode current of $17 \mathrm{nA}$ and a bias voltage of $1000 \mathrm{~V}$. The dynode pulse maximum is $\sim 1.8 \mathrm{~V}$, and the anode is $\sim 247.6 \mathrm{mV}$. The dynode/anode ratio is $\sim 7.3$, showing that the PMT amplifies $\sim 2.7$ times the current flowing from the last dynode to the anode.

The PMT and electronics readout must have a linear response to guarantee an accurate estimation of the energy deposited by the particles crossing the WCD. The linearity of the model was evaluated by correlating the dynode and anode pulse amplitude for different photocathode currents, and bias voltages [21]. Fig. 3 reproduces the dynode $v s$ anode amplitude for photocathode currents ranging 1-20 nA for $V_{B}=1000 \mathrm{~V}$ (red-line) and $V_{B}=900 \mathrm{~V}$ (black-line). The linear response of the PMT breaks down when it reaches its electrical limit at $1800 \mathrm{~V}\left(\sim 3 \times 10^{7}\right.$ gain $)$, causing a saturation effect in the pulse amplitude. 


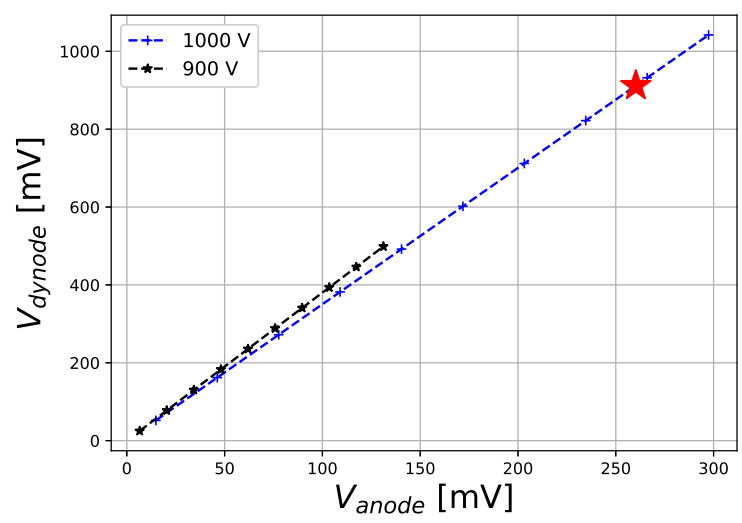

Figure 3: Correlation between the pulse maximum measured on the dynode and the anode at $1000 \mathrm{~V}$ (blue-line) and $900 \mathrm{~V}$ (black-line) for photocathode currents ranging 1-20 nA. The distance between data points indicates the gain increase depending on the bias voltage but preserving the response linearity. The star indicates the signal amplitude for a vertical muon.

\section{Conclusions}

We presented a PMT model which enhances the generalization of PMT Spice models, unlike others where the inter-dynode bias fraction is constant [9], limiting the model only to uniform voltage-dividers. Our approach allows using different voltage dividers (i.e. uniform or tapered) in photomultiplier tube simulations by configuring the bias voltage fraction in each inter-dynode stage.

The model implementation is not limited to Spice-based software but can also be used with compiled and interpreted programming languages. Particle and radiation detectors can implement this PMT model in their simulation frameworks to better understand the electronics frontend response.

\section{Acknowledgements}

The LAGO Collaboration is very thankful to the Pierre Auger Collaboration for their continuous support. It is also acknowledged the support of the LAGO Collaboration members.

\section{References}

[1] C. Sarmiento-Cano and the LAGO Collaboration, The Latin American Giant Observatory (LAGO) capabilities for detecting Gamma Ray Bursts, in 37th ICRC, no. 929, (Berlin, Germany), pp. 1-4, 2021.

[2] M. Suárez-Durán and the LAGO Collaboration, The LAGO space weather program: Directional geomagnetic effects, background fluence calculations and multi-spectral data analysis, in PoS(ICRC2015), Sissa Medialab, Aug., 2016, DOI.

[3] H. Asorey et al., Preliminary results from the Latin American Giant Observatory space weather simulation chain, Space Weather 16 (2018) 461. 
[4] R. de Aguiar, A. Fauth and the LAGO Collaboration, Forbush decrease on September 6-13, 2017 observed by the Tanca water-Cherenkov detector, in 37th ICRC, no. 1267, (Berlin, Germany), pp. 1-4, 2021.

[5] S. Vernetto, Detection of gamma-ray bursts in the $1 \mathrm{GeV}-1 \mathrm{TeV}$ energy range by ground-based experiments, Astroparticle Physics 13 (2000) 75.

[6] H. Asorey et al., Hardware-level calibration of the Chitaga water cherenkov detector in the GUANE array for space weather study, Scientia et technica 23 (2018) 563.

[7] X. Bertou et al., Calibration of the surface array of the Pierre Auger Observatory, Nucl. Instrum. Methods. Phys. Res. A 568 (2006) 839.

[8] A. Galindo et al., Calibration of a large water-cherenkov detector at the Sierra Negra site of LAGO, Nucl. Instrum. Methods. Phys. Res. A 861 (2017) 28.

[9] N. Krihely, SPICE model of photomultiplier tube under different bias conditions, IEEE Sensors Journal 14 (2014) 3606.

[10] D.Y. Akimov et al., Computer modelling of the hamamatsu r11410-20 PMT, Journal of Physics: Conference Series 798 (2017) 012211.

[11] F. Hueso-González et al., Comments on "spice model of photomultiplier tube under different bias conditions", IEEE Sensors Journal (2021) 1.

[12] A. Shih et al., Secondary electron emission studies, Applied Surface Science 111 (1997) 251.

[13] Hamamatsu Photonics K. K., Iwata City, Shizuoka Pref., Large photocathode area photomultiplier tubes, 2019.

[14] W.P. Huang et al., Design of the photomultiplier tube bases for high dynamic range readout in WCDA, Chinese Physics C 37 (2013) 036001.

[15] Hamamatsu Photonics K. K., Iwata City, Shizuoka Pref., Photomultiplier tubes: Basics and Applications, 2007.

[16] A. Vásquez-Ramírez et al., Simulated response of MuTe, a hybrid muon telescope, Journal of Instrumentation 15 (2020) P08004.

[17] R. Calderón et al., Geant4 based simulation of the water cherenkov detectors of the LAGO project, Nuclear and Particle Physics Proceedings 267-269 (2015) 424.

[18] Hamamatsu Photonics K. K., Iwata City, Shizuoka Pref., Photomultiplier tube R5912, 1998.

[19] M.S. Haro et al., The data acquisition system of the latin american giant observatory (LAGO), Nucl. Instrum. Methods. Phys. Res. A 820 (2016) 34.

[20] J. Peña-Rodríguez et al., Design and construction of MuTe: a hybrid muon telescope to study colombian volcanoes, Journal of Instrumentation 15 (2020) P09006.

[21] H. Arnaldi, Estudio de la señal de salida y diseño de la red de polarización de tubos fotomultiplicadores, Revista elektron 3 (2019) 112. 


\section{Full Authors List: LAGO Collaboration}

V. Agosín ${ }^{20}$, A. Alberto ${ }^{3}$, C. Alvarez ${ }^{16}$, J. Araya ${ }^{20}$, R. Arceo ${ }^{16}$, O. Areso ${ }^{13}$, L. H. Arnaldi ${ }^{2}$, H. Asorey ${ }^{14,7}$, M. Audelo ${ }^{9}$, M.G. Ballina-Escobar $^{19}$, D. C. Becerra-Villamizar ${ }^{18}$, X. Bertou ${ }^{2}$, K.S. Caballero-Mora ${ }^{16}$, R. Caiza ${ }^{8}$, R. Calderón-Ardila ${ }^{14}$, Calle, J. ${ }^{24}$, A. C. Fauth ${ }^{27}$, E. Carrera Jarrin ${ }^{26}$, L. E. Castillo Delacroix ${ }^{11}$, C. Castromonte ${ }^{25}$, Cazar-Ramírez D. ${ }^{26}$, Diego Cogollo ${ }^{28}$, D. A. Coloma Borja $^{26}$, R. Conde ${ }^{1}$, J. Cotzomi ${ }^{1}$, D. Dallara ${ }^{11}$, S. Dasso ${ }^{13,5,6}$, R. Aguiar ${ }^{27}$, Albuquerque, A. ${ }^{28}$, J.H.A.P.Reis ${ }^{27}$, H. De León ${ }^{16}$, R. deLeón-Barrios ${ }^{23}$, D. Domínguez ${ }^{8}$, M. Echiburu ${ }^{21}$, M. González ${ }^{2}$, M. Gómez Berisso ${ }^{2}$, J. Grisales Casadiegos ${ }^{23}$, A. M. Gulisano ${ }^{13,12,6}$, Juan Carlos Helo ${ }^{17}$, Condori, C. A. H. ${ }^{24}$, J. E. Ise ${ }^{11}$, Nascimento, G. K. M ${ }^{28}$, M. A. Leigui de Oliveira ${ }^{29}$, F. L. Miletto ${ }^{27}$, V. P. Luzio ${ }^{29}$, F. Machado ${ }^{25}$, Juan F. Mancilla-Caceres ${ }^{22}$, D. Manriquez ${ }^{20}$, A. Martínez-Méndez ${ }^{23}$, O. Martinez ${ }^{1}$, R. Mayo-García ${ }^{3}$, L.G. Mijangos ${ }^{22}$, Miranda, $\mathrm{P}^{24}$, M. G. Molina ${ }^{11}$, I.R. Morales ${ }^{19}$, O.G Morales-Olivares ${ }^{16}$, E. Moreno-Barbosa ${ }^{1}$, P. Muñoz ${ }^{17}$, Nina, $C^{24}$, L.A. Núñez ${ }^{23}$, L. Otininano ${ }^{4}$, R. Pagán-Muñoz ${ }^{3}$, K. M. Parada-Jaime ${ }^{18}$, H. M. Parada-Villamizar ${ }^{18}$, R. Parra $^{10}$, J. Peña-Rodríguez ${ }^{23}$, M. Pereira ${ }^{13}$, Y. A. Perez-Cuevas ${ }^{18}$, H. Perez ${ }^{19}$, J. Pisco-Guabave ${ }^{23}$, Raljevic, M. $^{24}$, M. Ramelli ${ }^{13}$, C. Ramírez ${ }^{22}$, Rivera, $\mathrm{H}^{24}$, L. T. Rubinstein ${ }^{13}$, A.J. Rubio-Montero $^{3}$, J.R. Sacahui ${ }^{19}$, H. Salazar ${ }^{1}$, N. Salomón ${ }^{11}$, J. Samanes ${ }^{4}$, N.A. Santos ${ }^{5}$, C. Sarmiento-Cano ${ }^{14}$, I. Sidelnik ${ }^{2}$, Mayra B. $\mathrm{Silva}^{22}$, O. Soto ${ }^{17}$, M. Suárez-Durán ${ }^{18,31}$, Subieta Vasquez, M. ${ }^{24}$, Terrazas C. ${ }^{24}$, Ticona, R. ${ }^{24}$, T. Torres Peralta ${ }^{11}$, Pablo A. Ulloa ${ }^{17}$, Z.R. Urrutia ${ }^{22}$, N. Vásquez ${ }^{8}$, A. Vázquez-Ramírez ${ }^{23}$, A. Vega ${ }^{20}$, P. Vega ${ }^{17}$, J. Vega ${ }^{4}$, A. Vesga-Ramirez ${ }^{14}$, D. Vitoreti ${ }^{30}$, R. Wiklich Sobrinho ${ }^{29}$,

${ }^{1}$ Benemérita Universidad Autónoma de Puebla". ${ }^{2}$ Centro Atómico Bariloche (CNEA/CONICET/IB)". ${ }^{3}$ CIEMAT". ${ }^{4}$ Comisión Nacional de Investigación y Desarrollo Aeroespacial". ${ }^{5}$ Departamento de Ciencias de la Atmósfera y los Océanos, Facultad de Ciencias Exactas y Naturales, Universidad de Buenos Aires.". ${ }^{6}$ Departamento de Física (FCEN,UBA)". ${ }^{7}$ Departamento Física Médica, CNEA-CONICET-UNSAM". ${ }^{8}$ Escuela Politécnica Nacional". ${ }^{9}$ Escuela Superior Politécnica de Chimborazo". ${ }^{10}$ European Soutern Observatory (ESO)". ${ }^{11}$ Facultad de Ciencias Exactas y Tecnología (FACET) - Universidad Nacional de Tucumán (UNT)". ${ }^{12}$ Instituto Antártico Argentino, Dirección Nacional del Antartico, Instituto de Astronomía y Física del Espacio (UBA-CONICET)". ${ }^{13}$ Instituto de Astronomía y Física del Espacio, IAFE (UBA-CONICET)". ${ }^{14}$ Instituto de Tecnologías en Detección y Astropartículas (CNEA, CONICET,UNSAM)". ${ }^{16}$ Universidad Autónoma de Chiapas". ${ }^{17}$ Universidad de La Serena". ${ }^{18}$ Universidad de Pamplona". ${ }^{19}$ Universidad de San Carlos". ${ }^{20}$ Universidad de Valparaíso". ${ }^{21}$ Universidad de Viña del Mar". ${ }^{22}$ Universidad del Valle de Guatemala". ${ }^{23}$ Universidad Industrial de Santander". ${ }^{24}$ Universidad Mayor de San Andrés". ${ }^{25}$ Universidad Nacional de Ingeniería". ${ }^{26}$ Universidad San Francisco de Quito". ${ }^{27}$ Universidade Estadual de Campinas". ${ }^{28}$ Universidade Federal de Campina Grande". ${ }^{29}$ Universidade Federal do ABC". ${ }^{30}$ Universidade Federal do Recôncavo da Bahia". ${ }^{31}$ Université Libre de Bruxelles, Brussels, Belgium.". 